\title{
APLICAÇÃO DE BACKSTEPPING PARA CONTROLE DE ÓXIDOS DE NITROGÊNIO EM VEÍCULOS DIESEL
}

\author{
Fabio L. Baldissera* \\ baldissera@das.ufsc.br \\ Ubirajara F. Moreno* \\ moreno@das.ufsc.br
}

\author{
Eugênio B. Castelan* \\ eugenio@das.ufsc.br
}

\author{
Edson R. De Pieri* \\ edson@das.ufsc.br
}

*Grupo de Controle de Sistemas Mecatrônicos (CSM), DAS/UFSC, Florianópolis (S.C.), Brasil

\begin{abstract}
Application of Backstepping Control to SCR Aftertreatment Systems

This paper presents the results concerning the application of a backstepping based control law to urea-SCR (selective calalytic reduction) aftertreatment systems in diesel engines. The devised control law is applied over a reduced order model for the SCR catalyst, which is effective for closed-loop control and, besides that, can be embedded in diesel electronic control units. The performance of the controller is evaluated in computer simulations by means of static and dynamic testing cycles, both for nominal and disturbed system's parameters.
\end{abstract}

KEYWORDS: SCR-aftertreatment, reduced order models, backstepping.

\section{RESUMO}

Este artigo apresenta a aplicação de uma técnica de controle do tipo backstepping a sistemas de pós-tratamento de gases de escape em veículos diesel operando com catalisadores SCR (selective catalytic reduction). A lei de controle é projetada para um modelo reduzido do sistema SCR, adequado ao controle em malha fechada e também à implementação nas unidades de controle ele-

\footnotetext{
Artigo submetido em 11/01/2011 (Id.: 1245)

Revisado em 28/05/2011, 19/09/2011, 29/10/2011

Aceito sob recomendação do Editor Associado Prof. Luis Fernando Alves
} Pereira trônico. O desempenho dessa lei de controle é avaliado em simulações computacionais por meio de ciclos de testes estático e dinâmico, tanto para situações em que os parâmetros de entrada assumem valores nominais, quanto para aquelas em que esses parâmetros apresentam perturbações.

PALAVRAS-CHAVE: Pós-Tratamento, sistemas SCR, modelos reduzidos, backstepping.

\section{INTRODUÇÃO}

A utilização de sistemas de pós-tratamento de gases de escape com catalisadores $\mathrm{SCR}^{1}$ (selective catalytic reduction) é uma das alternativas utilizadas atualmente para atingir os níveis legais de emissões de poluentes em veículos diesel. Essa tecnologia permite uma redução significativa na emissão dos óxidos de nitrogênio, $\mathrm{NO}_{\mathrm{x}}$, um dos principais poluentes de motores diesel (Seher et al., 2003). A redução na quantidade de $\mathrm{NO}_{\mathrm{x}}$ emitida pelo motor acontece pela injeção de uma solução aquosa de uréia na entrada do catalisador SCR, de forma que a amônia derivada desta reaja com os óxidos de nitrogênio gerados pelo motor, convertendo-os em nitrogênio, $\mathrm{N}_{2}$, e oxigênio, $\mathrm{O}_{2}$. Muita uréia injetada resultará em níveis indesejados de amônia na saída do catalisador;

\footnotetext{
${ }^{1}$ De fato, o que se denomina catalisador SCR na indústria automotiva é um reator catalítico. Pela consagração do uso, no entanto, utilizaremos a expressão catalisador SCR ao longo do texto.
} 
pouca uréia, por outro lado, terá eficácia reduzida na conversão dos óxidos de nitrogênio.

Atualmente, os sistemas comerciais baseados em catalisadores SCR utilizam predominantemente técnicas de controle em malha aberta, em geral, variantes da estratégia apresentada por Seher et al. (2003). A necessidade, no entanto, de atender às recentes e mais estritas leis de emissões de gases prejudiciais, garantir robustez do sistema ao longo do tempo e de, ao mesmo tempo, reduzir a complexidade de calibração dos sistemas comercializados motiva o estudo do controle em malha fechada baseado em modelos matemáticos.

No caso de sistemas SCR, as técnicas de controle em malha fechada podem ser divididas primariamente em dois grandes grupos: a) aquele que utiliza para realimentação o sinal vindo de um sensor de amônia, $\mathrm{NH}_{3}$, posicionado na saída do catalisador SCR (Wang et al., 2008; Willems et al., 2007); e b) aquele que se vale de um sensor de $\mathrm{NO}_{\mathrm{x}}$ após o catalisador (Devarakonda et al., 2008b; Chi and DaCosta, 2005; Willems et al., 2007; Schr et al., 2006).

A vantagem em fechar a malha do sistema com sensores de $\mathrm{NO}_{\mathrm{x}}$ decorre do fato de que esses sensores estarão presentes em configurações de pós-tratamento baseadas em SCR, como parte obrigatória do sistema de diagnose a bordo (OBD, do inglês on-board diagnostic).

Além da diferenciação quanto ao sinal usado na realimentação, as técnicas de controle em malha fechada disponíveis na literatura distinguem-se bastante quanto à modelagem do sistema SCR e, consequentemente, quanto à lei de controle mais adequada para o modelo adotado. Como mostraremos na seção de modelagem matemática desses sistemas, existem diversos fenômenos físicos e químicos envolvidos na operação dos catalisadores SCR, sendo que a análise detalhada de cada um é demasiadamente complexa. Portanto, um modelo matemático reduzido, mas ainda assim representativo o suficiente da realidade, também é um critério-chave para o sucesso do desempenho dos sistemas de pós-tratamento baseados em catalisadores SCR.

Em nosso trabalho, utilizaremos um sistema de equações diferenciais com quatro variáveis de estado como modelo para o catalisador. O estudo da aplicabilidade de tal modelo de ordem reduzida para fins de controle em malha fechada foi relatado no artigo de Devarakonda et al. (2008a). Este modelo é uma extensão de estudos anteriores baseados em sistemas com três variáveis de estado (Upadhyay and Nieuwstadt, 2006). Sobre esse modelo, usaremos uma estratégia de controle do tipo backstepping, técnica já largamente utilizada em aplicações envolvendo sistemas não-lineares, como sistemas pneumáticos de posicionamento e manipuladores robóticos, por exemplo (Ramirez et al., 2003; Guenther and Perondi, 2004). A escolha do backstepping deve-se a dois motivos: a) o sistema é não-linear e não opera em torno de um ponto de operação que enseje linearização; e b) a estrutura do sistema é inerentemente adequada ao uso da técnica, como será visto na seção 4 .

Para adequada compreensão dos objetivos deste trabalho, o artigo está organizado da seguinte forma: na seção 2, faremos uma rápida exposição do tema de emissão de poluentes. Na seção 3, apresentaremos a modelagem matemática de catalisadores SCR para, então, na seção 4, projetar uma lei de controle em malha fechada do tipo backstepping para sistemas de pós-tratamento que operam sobre tais catalisadores. Já na seção 5 , avaliaremos o desempenho do controlador quando variações são introduzidas na quantidade de óxidos de nitrogênio que entram no catalisador, $\mathrm{NO}_{\mathrm{x}, \mathrm{in}}$. Finalmente, na seção 6 , apresentaremos as conclusões e recomendações para trabalhos futuros.

\section{EMISSÃO DE GASES POLUENTES EM VEÍCULOS DIESEL}

É crescente, nos últimos anos, a demanda da sociedade por processos mais limpos de conversão de energia, de forma que os subprodutos da atividade humana em geral causem o menor impacto possível no meio ambiente. Essa exigência implica uma grande pressão por inovações no setor automotivo, haja vista que os veículos são responsáveis por grande parte das emissões antropogênicas de gases como óxidos de nitrogênio e monóxidos de carbono, por exemplo. Como medidas para redução da emissão dos gases poluentes advindos de veículos automotores, podemos citar em lista não exaustiva:

- viabilizar o transporte público e a utilização de outras formas de locomoção (ex.: bicicletas) nas grandes aglomerações urbanas;

- melhorar a qualidade dos combustíveis utilizados (ex.: nível de enxofre no diesel);

- investir em tecnologias para diminuir a chamada emissão crua dos motores à combustão interna, proveniente diretamente do pórtico de exaustão do motor (ex.: aperfeiçoando sistemas de injeção, geometria do motor, sistemas de partida e arrefecimento, bem como sistemas de admissão de ar);

- desenvolver sistemas automotivos de tração híbrida (ex.: diesel-elétrico) ou não dependentes de combustíveis fósseis (ex.: elétrico); 
- disponibilizar tecnologias de pós-tratamento dos gases de escape, de forma que a emissão crua dos motores seja reduzida pela ação subsequente de filtros e sistemas catalíticos (ex.: filtro de particulados, catalisadores de oxidação e catalisadores SCR, no caso de veículos diesel). Neste tópico, indicamos os trabalhos de Seher et al. (2003) e Schr et al. (2006), bem como as referências ali contidas, para maiores detalhes.

Concentraremo-nos, neste artigo, na última das medidas citadas e, especificamente, para o caso de sistemas catalíticos SCR aplicados a veículos diesel. Para compeendêla melhor, é necessário delinear alguns conceitos básicos da formação de poluentes em motores diesel, o que será feito a seguir.

Os poluentes mais significativos gerados pela combustão em motores diesel são os óxidos de nitrogênio, $\mathrm{NO}_{\mathrm{x}}$, e o material particulado, PM (do inglês particulate matter), esse último consistindo basicamente em fuligem associada a compostos orgânicos e enxofre (Heywood, 1988). A formação desses poluentes é tal que calibrações de motores diesel que privilegiam uma redução da emissão de óxidos de nitrogênio, para um dado ponto de operação do motor, apresentam maiores níveis de emissão de particulados e vice-versa (Heywood, 1988). O nível de emissão de material particulado e óxidos de nitrogênio vai depender especialmente das características da mistura ar-combustível, como: pressão, início e duração da injeção de diesel; geometria dos pórticos de entrada e exaustão de ar, bem como geometria da câmara de combustão do motor e massa de ar disponível na câmara de combustão (Raatz et al., 2005).

É importante ressaltarmos que a emissão de poluentes é afetada também pela rotação e torque do motor diesel, variáveis que caracterizam o ponto de operação do motor. Um detalhamento maior sobre formação e controle de poluentes pode ser encontrado nos livros de Guzzella and Onder (2004) e Heywood (1988).

A regulamentação dos limites para emissões dos poluentes é feita por diferentes órgãos, com ação de âmbito regional, espalhados pelo mundo. No Brasil, a CETESB (Companhia Ambiental do Estado de São Paulo) é o órgão técnico conveniado pelo IBAMA (Instituto Brasileiro do Meio Ambiente e dos Recursos Naturais Renováveis) para implantação e operacionalização do Programa de Controle de Poluição do Ar por Veículos Automotores, o Proconve.

A fim de medir o nível de emissão de poluentes dos motores diesel, ciclos de testes padronizados são utilizados.
Esses ciclos procuram simular as condições de torque e velocidade encontradas pelos veículos em seus respectivos países. Neste trabalho serão empregados os ciclos de testes ESC (European Static Cycle) e ETC (European Transient Cycle), ambos utilizados para avaliar as emissões das legislações européias de EURO III a EURO V. Informações consolidadas sobre as diferentes legislações (e respectivas especificações) podem ser encontradas na página eletrônica www.dieselnet.com/standards, fórum mantido por empresas automotivas e centros de pesquisa em motores diesel e que abriga artigos e informações técnicas sobre assuntos correlatos.

\section{SISTEMA DE PÓS-TRATAMENTO COM CATALISADORES SCR}

\subsection{Visão Geral}

Nos sistemas baseados em catalisadores SCR, os óxidos de nitrogênio gerados pelo motor são reduzidos através da reação com amônia, $\mathrm{NH}_{3}$, no interior do catalisador SCR. A amônia é obtida pela injeção de uma solução aquosa de 32,5\% de uréia (conhecida comercialmente como Adblue) na entrada do catalisador SCR, como mostra esquematicamente a figura 1. A água da solução evapora devido às altas temperaturas do gás de escape e, em sequência, a uréia passa por estágios de decomposição térmica e hidrólise, gerando $\mathrm{NH}_{3}$ e $\mathrm{CO}_{2}$ ao final de todo o processo. Somente $50 \%$ da amônia fica disponível na entrada do catalisador, pois a hidrólise é uma reação que precisa ser catalisada, só finalizando por completo no interior do SCR (Willems et al., 2007). A solução aquosa de uréia não é tóxica e sua obtenção é simples, dada a larga utilização da uréia em diversos setores da atividade humana (ex.: produção de cosméticos e de fertilizantes agrícolas).

Além da comprovada eficácia na redução de óxidos de nitrogênio, a utilização de sistemas SCR possibilita, ainda, calibrar o motor diesel de forma a reduzir o consumo de combustível (Seher et al., 2003; Schr et al., 2006).

\subsection{Modelagem Matemática}

As três principais reações químicas que acontecem no interior de um catalisador SCR para conversão dos óxidos de nitrogênio são descritas pelas equações (1), (2) e (3), mostradas a seguir, também conhecidas como reações rápida, padrão e lenta, respectivamente:

$$
\begin{aligned}
& 4 \mathrm{NH}_{3}+2 \mathrm{NO}+2 \mathrm{NO}_{2} \rightarrow 4 \mathrm{~N}_{2}+6 \mathrm{H}_{2} \mathrm{O} \\
& 4 \mathrm{NH}_{3}+4 \mathrm{NO}+\mathrm{O}_{2} \rightarrow 4 \mathrm{~N}_{2}+6 \mathrm{H}_{2} \mathrm{O} \\
& 8 \mathrm{NH}_{3}+6 \mathrm{NO}_{2} \rightarrow 7 \mathrm{~N}_{2}+12 \mathrm{H}_{2} \mathrm{O}
\end{aligned}
$$




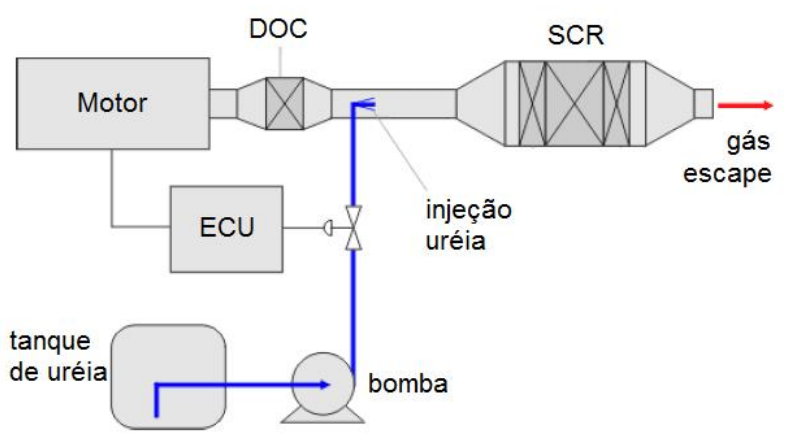

Figura 1: Representação esquemática de um sistema comercial de pós-tratamento do tipo SCR. DOC representa o catalisador de oxidação (diesel oxidation catalyst) e ECU a unidade de comando eletrônico (electronic control unit). Figura baseada no artigo de Majewski (2005).

A amônia injetada, além de reagir com os óxidos de nitrogênio, também é oxidada no interior do catalisador SCR segundo a equação

$$
4 \mathrm{NH}_{3}+3 \mathrm{O}_{2} \rightarrow 2 \mathrm{~N}_{2}+3 \mathrm{H}_{2} \mathrm{O} .
$$

Não consideraremos oxidação da amônia em óxidos de nitrogênio, pois estudos reportam que catalisadores SCR do tipo Fe-zeolite apresentam $100 \%$ de seletividade de oxidação de amônia para nitrogênio em temperaturas inferiores a $T=600^{\circ} \mathrm{C}$ (Devarakonda et al., 2008a), limite esse que não será excedido em nossos experimentos.

Os fenômenos de adsorção e dessorção de amônia também serão incluídos em nosso modelo matemático, representados, respectivamente, pelas equações:

$$
\begin{aligned}
& \mathrm{NH}_{3} \rightarrow \mathrm{NH}_{3}^{*} \\
& \mathrm{NH}_{3}^{*} \rightarrow \mathrm{NH}_{3}
\end{aligned}
$$

em que $\mathrm{NH}_{3}^{*}$ indica a amônia adsorvida pelo catalisador. A figura 2 mostra esquematicamente os diferentes fenômenos que acontecem no interior do catalisador, responsáveis pela ocorrência das reações descritas nas equações (1)-(6).

As taxas de ocorrência $R_{i}$ das seis reações químicas anteriormente apresentadas são calculadas pelas expressões (Devarakonda et al., 2008a):

$$
\begin{aligned}
& R_{1}=k_{1} C_{\mathrm{NO}} C_{\mathrm{NO}_{2}} \theta \Omega \\
& R_{2}=k_{2} C_{\mathrm{NO}} \mu_{\mathrm{O}_{2}} \theta \Omega \\
& R_{3}=k_{3} C_{\mathrm{NO}_{2}} \theta \Omega \\
& R_{4}=k_{4} \theta \Omega \\
& R_{5}=k_{5}(1-\theta) C_{\mathrm{NH}_{3}} \Omega \\
& R_{6}=k_{6} \theta \Omega
\end{aligned}
$$

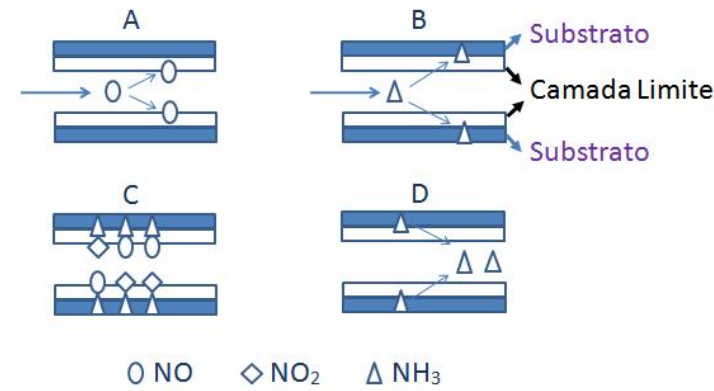

Figura 2: Os quatro principais fenômenos físicos e químicos que ocorrem no interior do catalisador. A: difusão dos gases na camada limite; B: adsorção de amônia no substrato do catalisador; C: reação química dos óxidos de nitrogênio com amônia; e D: dessorção da amônia. Figura baseada no trabalho de Devarakonda et al. (2008a).

em que $C_{i}$ denota a concentração da espécie $i$ no interior do $\mathrm{SCR}\left(\mathrm{em} \mathrm{mol} / \mathrm{m}^{3}\right) ; \theta$ representa a fração de cobertura do catalisador por amônia, definida como a razão do número de sítios do catalisador preenchidos com amônia sobre o número total de sítios existentes; $\Omega$ é a capacidade total de adsorção de amônia pelo catalisador, medida em $\mathrm{mol} / \mathrm{m}^{3}$ de gás de escape, e $\mu_{\mathrm{O}_{2}}$ simboliza a fração molar de oxigênio no gás de escape (em \%). Os coeficientes de velocidade de conversão das reações, $k_{i}$, são definidos pela equação de Arrhenius:

$$
k_{i}(T)=A_{i} e^{-\frac{E_{i}}{R T}}(i=1 \ldots 6)
$$

sendo $A_{i}$ um fator pré-exponencial; $E_{i}$, a energia de ativação da reação $i(\mathrm{em} \mathrm{J} / \mathrm{mol}) ; R$ é a constante universal dos gases, medida em $\mathrm{J} /(\mathrm{mol} \times \mathrm{K})$, e $\mathrm{T}$, a temperatura.

Definidas as reações químicas e a cinética de suas ocorrências, o sistema de equações diferenciais reduzido que utilizaremos como base para o projeto da lei de controle em malha fechada do sistema SCR é descrito, então, como (Devarakonda et al., 2008a):

$$
\begin{aligned}
\dot{C}_{\mathrm{NO}}= & -Q C_{\mathrm{NO}}-k_{1} \Omega \theta C_{\mathrm{NO}} C_{\mathrm{NO}_{2}}-k_{2} \Omega \theta C_{\mathrm{NO}} \mu_{\mathrm{O}_{2}} \\
& +Q C_{\mathrm{NO}, \mathrm{in}} \\
\dot{C}_{\mathrm{NO}_{2}}= & -Q C_{\mathrm{NO}_{2}}-k_{1} \Omega \theta C_{\mathrm{NO}} C_{\mathrm{NO}_{2}}-k_{3} \Omega \theta C_{\mathrm{NO}_{2}} \\
& +Q C_{\mathrm{NO}_{2}, \text { in }} \\
\dot{\theta}= & -\left(k_{6}+k_{4}\right) \theta+k_{5} C_{\mathrm{NH}_{3}}-k_{1} C_{\mathrm{NO}} C_{\mathrm{NO}_{2}} \theta \\
& -k_{2} C_{\mathrm{NO}_{2}} \mu_{\mathrm{O}_{2}} \theta-k_{3} C_{\mathrm{NO}_{2}} \theta-k_{5} \theta C_{\mathrm{NH}_{3}} \\
\dot{C}_{\mathrm{NH}_{3}}= & +k_{6} \Omega \theta-\left(k_{5} \Omega+Q\right) C_{\mathrm{NH}_{3}}+k_{5} \Omega \theta C_{\mathrm{NH}_{3}} \\
& +Q C_{\mathrm{NH}_{3}, \text { in }}
\end{aligned}
$$

em que $C_{i, i n}$ representa a concentração da espécie $i$ na entrada do catalisador e $Q$ denota a vazão volumétrica 
de gás de escape, $Q^{\prime}$, normalizada pelo volume $V$ do catalisador, ou seja, $Q=Q^{\prime} / V$. A variável $Q$ é também conhecida como velocidade espacial (space velocity), SV, do gás no interior do catalisador.

Entre as principais hipóteses utilizadas para obtenção do sistema de equações (8) estão (Devarakonda et al., 2008a):

1. A concentração $C_{i}$ das espécies no interior do catalisador, a velocidade e a temperatura dos gases de escape são uniformes, transversal e longitudinalmente, no SCR;

2. A temperatura do gás de escape utilizada nas equações é calculada como a média da temperatura dos gases na entrada e saída do catalisador;

3. Os gases são ideais;

4. A evaporação, decomposição e hidrólise da uréia injetada ocorrem completamente na entrada do catalisador. Assim, cada mol de uréia injetada no duto de escape formará dois moles de $\mathrm{NH}_{3}$ na entrada do catalisador e estará disponível inteiramente para redução de $\mathrm{NO}_{\mathrm{x}}$;

5. Quaisquer reações colaterais envolvendo a formação de $\mathrm{N}_{2} \mathrm{O}$ e $\mathrm{NH}_{4} \mathrm{NO}_{3}$ no interior do catalisador são desprezadas.

O modelo matemático aqui descrito, baseado nas hipóteses apresentadas, capta os principais fenômenos que acontecem no interior de um catalisador SCR e foi validado em bancada de motores, mostrando-se adequado para controle em malha fechada (Devarakonda et al., 2008a). Uma análise matemática mais detalhada de sistemas derivados de reações químicas (lei de ação das massas) pode ser encontrada no trabalho de Chellaboina et al. (2009).

Tomando como vetor das variáveis de estado $\left[\begin{array}{llll}x_{1} & x_{2} & x_{3} & x_{4}\end{array}\right]^{T}=\left[\begin{array}{llll}C_{\mathrm{NO}} & C_{\mathrm{NO}_{2}} & \theta & C_{\mathrm{NH}_{3}}\end{array}\right]^{T}, \quad$ o sistema de equações diferenciais (8) pode ser reescrito como:

$$
\begin{aligned}
\dot{x}_{1}= & -Q x_{1}-k_{1} \Omega x_{1} x_{2} x_{3}-k_{2} \Omega x_{1} x_{3} \mu+Q w_{1} \\
\dot{x}_{2}= & -Q x_{2}-k_{1} \Omega x_{1} x_{2} x_{3}-k_{3} \Omega x_{2} x_{3}+Q w_{2} \\
\dot{x}_{3}= & -\left(k_{4}+k_{6}\right) x_{3}+k_{5}\left(1-x_{3}\right) x_{4}-k_{3} x_{2} x_{3} \\
& -k_{1} x_{1} x_{2} x_{3}-k_{2} x_{1} x_{3} \mu \\
\dot{x}_{4}= & +k_{6} \Omega x_{3}-Q x_{4}-k_{5} \Omega\left(1-x_{3}\right) x_{4}+Q u
\end{aligned}
$$

sendo $u=\mathrm{C}_{\mathrm{NH}_{3, \text { in }}}$ a entrada de controle, ou seja, a quantidade de amônia injetada no duto de escape e $w=\left[\begin{array}{ll}w_{1} & w_{2}\end{array}\right]^{T}$ o vetor da concentração de óxidos de nitrogênio na entrada do catalisador SCR (ambas as variáveis medidas em $\mathrm{mol} / \mathrm{m}^{3}$ de gás de escape), com $w_{1}=\mathrm{C}_{\mathrm{NO}, \text { in }}$ e $w_{2}=\mathrm{C}_{\mathrm{NO}_{2, \text { in }}}$.

\section{CONTROLE DE SISTEMAS SCR}

\subsection{Abordagem via Backstepping}

Revisaremos brevemente a abordagem backstepping (Khalil, 2002; Pagano, 2007) quando utilizada para estabilizar a origem de sistemas representados pelas equações:

$$
\begin{aligned}
& \dot{\eta}=f(\eta)+g(\eta) \xi \\
& \dot{\xi}=f_{a}(\eta, \xi)+g_{a}(\eta, \xi) u
\end{aligned}
$$

em que $[\eta, \xi]^{T} \in R^{n+1}$ é o vetor de estados, $u \in R$, a entrada de controle e $f, g: D \rightarrow R^{n}$, com $f(0)=0$.

Se $g_{a}(\eta, \xi) \neq 0$ para o domínio de interesse, a transformação

$$
u=\frac{1}{g_{a}(\eta, \xi)}\left[u_{a}-f_{a}(\eta, \xi)\right]
$$

reduzirá a equação (11) ao integrador $\dot{\xi}=u_{a}$.

Seja $\phi(\eta), \phi(0)=0$, uma função tal que a origem da equação (10) seja estável para $\xi=\phi(\eta)$. Suponhamos, ainda, que conhecemos uma função de Lyapunov $V(\eta)$ satisfazendo

$$
\dot{V}(\eta)=\frac{\partial V}{\partial \eta}[f(\eta)+g(\eta) \phi(\eta)] \leq-W(\eta), \forall \eta \in D
$$

com $W(\eta)$ uma função definida positiva. Então, o controle

$$
\begin{aligned}
u= & \frac{1}{g_{a}(\eta, \xi)}\left\{\frac{\partial \phi}{\partial \eta}[f(\eta)+g(\eta) \xi]-\frac{\partial V}{\partial \eta} g(\eta)-\right. \\
& -k[\xi-\phi(\eta)]-f(\eta, \xi)\}
\end{aligned}
$$

estabiliza a origem do sistema de equações (10)-(11) para algum $k>0$, juntamente com a função de Lyapunov

$$
V_{c}(\eta, \xi)=V(\eta)+\frac{1}{2}[\xi-\phi(\eta)]^{2} .
$$

Daremos um passo adiante no processo da síntese de controladores por meio da técnica de backstepping, considerando agora sistemas estritamente realimentados (Khalil, 2002), que assumem a forma:

$$
\begin{aligned}
& \dot{x}=f_{0}(x)+g_{0}(x) z_{1} \\
& \dot{z}_{1}=f_{1}\left(x, z_{1}\right)+g_{1}\left(x, z_{1}\right) z_{2} \\
& \dot{z}_{2}=f_{2}\left(x, z_{1}, z_{2}\right)+g_{2}\left(x, z_{1}, z_{2}\right) u
\end{aligned}
$$


com $g_{i}\left(x, z_{1}, \ldots, z_{i}\right) \neq 0$ para $0 \leq i \leq 2$, no domínio de interesse $D$.

Iniciamos o procedimento recursivo, tomando o subsistema

$$
\begin{aligned}
& \dot{x}=f_{0}(x)+g_{0}(x) z_{1} \\
& \dot{z}_{1}=f_{1}\left(x, z_{1}\right)+g_{1}\left(x, z_{1}\right) z_{2}
\end{aligned}
$$

e consideremos que seja possível encontrar uma função $\phi_{0}(x)$, com $\phi(0)=0$, e uma função de Lyapunov $V_{0}(x)$, tais que:

$$
\frac{\partial V_{0}}{\partial x}\left[f_{0}(x)+g_{0}(x) \phi_{0}(x)\right] \leq-W(x)
$$

para o domínio de interesse e alguma função definida positiva $W(x)$. De posse de $\phi_{0}(x)$ e $V_{0}(x)$, fazendo $x=$ $\eta, g_{0}=g, z_{1}=\xi, f_{1}=f_{a}, g_{1}=g_{a}, z_{2}=u$ e utilizando as equações (14) e (15), obtemos uma lei de controle $z_{2}$ e uma função de Lyapunov, que estabilizam o subsistema considerado, dadas por

$$
\begin{aligned}
z_{2}= & \phi_{1}\left(x, z_{1}\right)=\frac{1}{g_{1}}\left[\frac{\partial \phi_{0}}{\partial x}\left(f_{0}+g_{0} z_{1}\right)-\frac{\partial V_{0}}{\partial x} g_{0}-\right. \\
& \left.-k_{1}\left(z_{1}-\phi_{0}\right)-f_{1}\right]
\end{aligned}
$$

$\operatorname{com} k_{1}>0 \mathrm{e}$

$$
V_{1}=V_{0}(x)+\frac{1}{2}\left[z_{1}-\phi_{0}(x)\right]^{2}
$$

Por último, consideramos o sistema formado pelos estados $\left[\begin{array}{lll}x & z_{1} & z_{2}\end{array}\right]^{T}$ do sistema de equações diferenciais (16) como um caso particular das equações (10)-(11), de forma a obter a lei de controle $u$ que estabiliza a origem do sistema (16) com a função de Lyapunov associada, dadas pelas equações (19) e (20), respectivamente

$$
\begin{aligned}
u= & \frac{1}{g_{2}}\left[\frac{\partial \phi_{1}}{\partial x}\left(f_{0}+g_{0} z_{1}\right)+\frac{\partial \phi_{1}}{\partial z_{1}}\left(f_{1}+g_{1} z_{2}\right)-\right. \\
& \left.-\frac{\partial V_{1}}{\partial z_{1}} g_{1}-k_{2}\left(z_{2}-\phi_{1}\right)-f_{2}\right]
\end{aligned}
$$

$\operatorname{com} k_{2}>0 \mathrm{e}$

$$
V_{2}=V_{1}\left(x, z_{1}\right)+\frac{1}{2}\left[z_{2}-\phi_{2}\left(x, z_{1}\right)\right]^{2}
$$

\subsection{Aplicação da Técnica Backstepping a Sistemas SCR}

O sistema de equações diferenciais (9) pode ser visto como um sistema estritamente realimentado com

$$
\begin{aligned}
x= & {\left[\begin{array}{l}
x_{1} \\
x_{2}
\end{array}\right], f_{0}=\left[\begin{array}{c}
-Q x_{1}+Q w_{1} \\
-Q x_{2}+Q w_{2}
\end{array}\right], z_{1}=x_{3} } \\
g_{0}= & {\left[\begin{array}{c}
-\left(k_{1} \Omega x_{1} x_{2}+k_{2} \Omega x_{1} \mu\right) \\
-\left(k_{1} \Omega x_{1} x_{2}+k_{3} \Omega x_{2}\right)
\end{array}\right], z_{2}=x_{4} } \\
f_{1}= & -\left(k_{4}+k_{6}\right) x_{3}-k_{3} x_{2} x_{3}-k_{1} x_{1} x_{2} x_{3} \\
& -k_{2} x_{1} x_{3} \mu \\
f_{2}= & +k_{6} \Omega x_{3}-Q x_{4}-k_{5} \Omega\left(1-x_{3}\right) x_{4} \\
g_{1}= & +k_{5}\left(1-x_{3}\right), g_{2}=Q .
\end{aligned}
$$

As funções $g_{i}$ assim definidas são diferentes de zero para $x_{1}, x_{2} \neq 0, x_{3} \neq 1$ e $Q \neq 0$, condições satisfeitas durante a operação do sistema de pós-tratamento. Se o motor está em funcionamento, $Q$ é sempre diferente de zero. A quantidade de óxidos de nitrogênio que deixam o catalisador, $x_{1}$ e $x_{2}$, nunca é nula, pois a conversão dos óxidos não é completa no interior do catalisador. Finalmente, devido ao fenômeno da dessorção térmica, o catalisador SCR nunca fica preenchido completamente de amônia, ou seja, $x_{3} \neq 1$ para todas as situações práticas.

\subsubsection{Objetivos de Controle}

O nosso objetivo de controle não é estabilizar a origem do sistema de equações diferenciais (16), mas sim: a) converter os óxidos de nitrogênio que entram no catalisador, $\left[w_{1}(t)+w_{2}(t)\right]$, de forma que os óxidos de nitrogênio que saem do catalisador, $\left[x_{1}(t)+x_{2}(t)\right]$, estejam dentro do limite permitido pela legislação de emissões vigente; e b) reduzir a amônia injetada e não reagida, $x_{4}$, também chamada de slip de amônia. O limite máximo de óxidos de nitrogênio esperado na saída do catalisador para os ciclos estático e dinâmico será de $2 \mathrm{~g} / \mathrm{kWh}$, limite esse que entrou em vigor no Brasil para veículos comerciais diesel em 2012. Para o slip de amônia, estabeleceremos $10 \mathrm{ppm}$ e $25 \mathrm{ppm}$ como valores máximos para a média e pico, respectivamente, nos ciclos de testes. Embora o nível de emissão de amônia não seja comumente regulamentado, valores de 10 ppm para média e 25 ppm para pico são geralmente utilizados (Willems et al., 2007) ${ }^{2}$.

Relaxarmos a restrição de estabilização da origem implica também abandonarmos as exigências de que $f(0)=$ $0, \phi_{0}(0)=0$ e a de que a desigualdade (13) seja satisfeita. No entanto, precisamos garantir que, mesmo com

\footnotetext{
${ }^{2} \mathrm{~A}$ legislação Euro VI define 10 ppm como limite médio no ciclo para emissão de $\mathrm{NH}_{3}$, sem qualquer menção aos valores de pico.
} 
o relaxamento dessas restrições, o uso de $u$ como definido pela equação (19), permita que: a) a taxa de variação da energia de $V_{0}(x)$ seja limitada e que, além disso, b) os erros $\left(z_{2}-\phi_{1}\right)$ e $\left(z_{1}-\phi_{0}\right)$ sejam limitados a valores tão pequenos quanto desejarmos. A convergência de $z_{i}$ para $\phi_{i-1}$ é importante, pois na técnica de backstepping a função $\phi_{0}$ é, em última instância, a responsável pelo desempenho do sistema de controle. São esses dois pontos que mostraremos serem válidos a seguir.

\subsubsection{Limite para a Taxa de Variação de $V_{0}(x)$}

Suponhamos $V_{0}\left(x_{1}, x_{2}\right)=\frac{1}{2} x_{1}^{2}+\frac{1}{2} x_{2}^{2}$ para o subsistema formado pelo estado $x=\left[\begin{array}{ll}x_{1} & x_{2}\end{array}\right]^{T}$ da equação (16), com $Q, x_{i}, w_{i}, z_{1}>0$. A taxa de variação de $V_{0}$ no tempo é dada por

$$
\begin{aligned}
\dot{V}_{0} & =\frac{\partial V_{0}}{\partial x} \frac{d x}{d t}=\left[x_{1} x_{2}\right]\left[\begin{array}{c}
f_{0}(1)+g_{0}(1) x_{3} \\
f_{0}(2)+g_{0}(2) x_{3}
\end{array}\right]< \\
& <\left[x_{1} x_{2}\right]\left[\begin{array}{l}
-Q x_{1}+Q w_{1} \\
-Q x_{2}+Q w_{2}
\end{array}\right]=-Q x^{T} x+Q x^{T} w .
\end{aligned}
$$

Veja que a primeira desigualdade é válida, pois $g_{0}(1) x_{3}$ e $g_{0}(2) x_{3}$ são estritamente negativos. E como $x_{i} \leq w_{i}$ (conservação de massa aplicada aos óxidos de nitrogênio), com $x_{i}, w_{i} \geq 0$, para $i=1,2$ :

$$
\dot{V}_{0}<-Q x^{T} x+Q x^{T} w \leq-Q x^{T} x+Q w^{T} w<Q w^{T} w .
$$

Portanto, $\dot{V}_{0}<Q w^{T} w=Q\|w\|_{2}$ para qualquer $z_{1}>0$, ou seja, a taxa de variação de $V_{0}$ é limitada superiormente pela energia do sinal de entrada dos óxidos de nitrogênio, $w(t)$, e pela velocidade espacial do gás de escape, $Q(t)$.

\subsubsection{Dinâmica do Sinal $e_{i}=z_{i}-\phi_{i-1}$}

Um limite superior para a taxa de variação da energia de nosso sistema por si só não basta. Para alcançarmos os objetivos desejados, é necessário que $z_{1}$, a quantidade de amônia adsorvida no interior do catalisador, esteja o mais próxima possível de $\phi_{0}(x)$. Demonstraremos que isso de fato acontece.

Seja $\phi_{0}$ uma função, tal que

$$
\dot{x}=f_{0}(x)+g_{0}(x) \phi_{0}
$$

tenha o comportamento esperado em termos de eficiência de conversão dos óxidos de nitrogênio no interior do catalisador com

$$
\dot{V}_{0}=\frac{\partial V_{0}}{\partial x}\left(f_{0}+g_{0} \phi_{0}\right)=P(x)<Q w^{T} w
$$

A dinâmica do sinal de erro $e_{1}=z_{1}-\phi_{0}$, ao tomarmos $\dot{z}_{1}$ como definida pelo sistema de equações diferenciais (16) e $z_{2}$ pela equação (17), pode ser escrita como:

$$
\begin{aligned}
& \dot{e}_{1}=\left(\dot{z}_{1}-\dot{\phi}_{0}\right)=f_{1}+g_{1} z_{2}-\dot{\phi}_{0} \\
& \dot{e}_{1}=-\frac{\partial V_{0}}{\partial x} g_{0}(x)-m_{1} e_{1} .
\end{aligned}
$$

Assim, podemos fazer o sinal $e_{1}$ tão pequeno quanto quisermos, quanto maior for o ganho $m_{1}$, um parâmetro de alto ganho do sistema de controle (Khalil, 2002). Raciocínio semelhante utilizamos para a dinâmica do sinal $e_{2}=z_{2}-\phi_{1}$, adotando $\dot{z}_{2}$ como definida pelo sistema de equações (16) e $u$ pela equação (19), obtendo, assim:

$$
\dot{e}_{2}=-\frac{\partial V_{1}}{\partial z_{1}} g_{1}-m_{2} e_{2} .
$$

\section{SIMULAÇÕES E RESULTADOS}

Vimos na seção anterior, que a técnica de backstepping pode ser utilizada mesmo quando a taxa de variação da função de Lyapunov $V_{0}$ assume valores positivos em determinados intervalos de tempo. Avaliaremos nesta seção o desempenho de uma lei de controle do tipo backstepping calculada conforme o procedimento descrito na subseção 4.1 para duas $\phi_{0}$ diferentes. Ressaltamos que a lei de controle do tipo backstepping fica determinada quando da especificação de $\phi_{0}$, por meio do uso das equações (17), (18) e (19).

Os resultados foram obtidos por meio da modelagem computacional do sistema de equações diferenciais (9), com parâmetros de um catalisador SCR de Fe-zeolite relatados por Devarakonda et al. (2008b). Os dados para alimentação do modelo, como concentrações de óxidos de nitrogênio, temperatura dos gases de escape, etc. são dados típicos de uma aplicação comercial para um motor diesel de 4.8 l, obtidos em dinamômetro para os ciclos de testes ESC e ETC, com a utilização de um catalisador de oxidação, DOC, na saída do motor (ver figura 1). As variáveis $x_{1}$ e $x_{2}$ são consideradas medidas através de um sensor de $\mathrm{NO}_{\mathrm{x}}$, ou seja, desprezaremos a sensibilidade cruzada dos sensores comerciais de $\mathrm{NO}_{\mathrm{x}}$ à amônia; as variáveis $x_{3}$ e $x_{4}$, por sua vez, são estimadas pelo sistema de equações diferenciais (9), que pode, neste caso, ser visto como o observador de estados em malha aberta do sistema (esta solução é comumente utilizada em sistemas de pós-tratamento comerciais).

Avaliaremos a robustez do controlador para um aumento de $30 \%$ na quantidade de $\mathrm{NO}_{\mathrm{x}, \mathrm{in}}$. Esse aumento simula os efeitos de variações nas emissões cruas de motores diesel, tipicamente encontradas na prática e causadas 
por fatores como umidade do ar ou envelhecimento do motor (Willems et al., 2007). Essas variações não são detectadas por um sistema em malha aberta, haja vista que, por motivos de custos, a quantidade de óxidos de nitrogênio gerada por um motor diesel geralmente não é medida, mas sim estimada por mapas de calibração.

\subsection{Utilização de $\phi_{0}$ Linear}

Tomemos, agora,

$$
\phi_{0}=p_{1}\left(x_{1}+x_{2}\right)+p_{2}\left(w_{1}+w_{2}\right)
$$

com $p_{1}$ e $p_{2}$ parâmetros calibráveis de tal forma que a conversão de $\mathrm{NO}_{\mathrm{x}}$ no catalisador esteja dentro do desejado, e que a quantidade de amônia não utilizada nas reações de conversão, $x_{4}=z_{2}$, ou slip de amônia, seja reduzida. Perceba que $\phi_{0}$ tem um apelo físico elementar: tanto maior será a cobertura do catalisador por amônia, $\phi_{0}$, quanto maior a quantidade de óxidos de nitrogênio que entram no catalisador $\mathrm{NO}_{\mathrm{x}, \mathrm{in}}$, ou quanto maior a quantidade de óxidos de nitrogênio não convertidos pelo catalisador, $\mathrm{NO}_{\mathrm{x}, \mathrm{out}}$.

A lei de controle final que utilizaremos é dada por $u_{\text {final }}=p_{x_{4}} u$, em que $u$ é calculado pela equação (19) e $p_{x_{4}}$ é um fator de saturação dependente do slip de amônia, e assume a forma

$$
p_{x_{4}}= \begin{cases}1, & \text { se } x_{4} \leq 10 \mathrm{ppm} \\ \frac{5}{3}-\frac{1}{15} x_{4}, & \text { se } 10 \mathrm{ppm}<x_{4} \leq 25 \mathrm{ppm} \\ 0, & \text { se } x_{4}>25 \mathrm{ppm}\end{cases}
$$

O objetivo de $p_{x_{4}}$ é manter os picos de slip de amônia abaixo de $25 \mathrm{ppm}$. Assim, para valores moderados de slip (em que moderado foi definido empiricamente como um valor entre 0 e $10 \mathrm{ppm}$ ), a lei de controle $u$ dada pela equação (19) não se altera. A partir de 10 ppm a lei de controle sofre uma progressiva redução até ser anulada quando o slip de amônia ultrapassa $25 \mathrm{ppm}$. Desta forma, $p_{x_{4}}$ compensa o fato de $\phi_{0}$ não ser uma função da temperatura do gás de escape (e, por não ser função da temperatura, não diminuir o nível desejado de amônia no interior do catalisador quando a temperatura aumenta, o que reduziria o fenômeno de dessorção e, portanto, o slip de amônia).

A tabela 1 mostra os resultados obtidos no ciclo ESC para diferentes pares $\left(p_{1}, p_{2}\right)$, escolhidos de forma que a conversão dos óxidos de nitrogênio seja a mesma para o caso nominal. Na tabela 1 colocamos também os resultados obtidos quando aplicamos uma técnica de controle em malha aberta, MA, baseada no trabalho de Seher et al. (2003).
Tabela 1: Resultados da simulação no ciclo ESC. $\mathrm{NO}_{\mathrm{x}, \text { in }}$ no caso nominal é de $12,03 \mathrm{~g} / \mathrm{kWh}$.

\begin{tabular}{cccc}
\hline \hline $\mathrm{NO}_{\mathrm{x}, \text { in }}$ & $\left(p_{1} ; p_{2}\right)$ & $\begin{array}{c}\mathrm{NO}_{\mathrm{x}, \text { out }} \\
{[\mathrm{g} / \mathrm{kWh}]}\end{array}$ & $\begin{array}{c}\text { slip } \mathrm{NH}_{3} \\
\text { médio } \\
{[\mathrm{ppm}]}\end{array}$ \\
\hline nominal & $\mathrm{MA}$ & 1,60 & 11,77 \\
& $(0,0 ; 7,5)$ & 1,60 & 8,27 \\
& $(8,2 ; 6,0)$ & 1,60 & 8,09 \\
& $(20 ; 4,0)$ & 1,60 & 8,00 \\
& $(32 ; 2,0)$ & 1,60 & 7,92 \\
& $(43 ; 0,0)$ & 1,60 & 7,82 \\
\hline $30 \%$ & $\mathrm{MA}$ & 2,45 & 7,69 \\
& $(0,0 ; 7,5)$ & 2,41 & 5,82 \\
& $(8,2 ; 6,0)$ & 2,34 & 6,13 \\
& $(20 ; 4,0)$ & 2,25 & 6,62 \\
& $(32 ; 2,0)$ & 2,19 & 7,01 \\
& $(43 ; 0,0)$ & 2,14 & 7,33 \\
\hline
\end{tabular}

À primeira vista, observamos a grande conversão de óxidos de nitrogênio que se torna possível com o uso de sistemas SCR: de 12,03 g/kWh que são gerados pelo motor, somente 1,60 g/kWh deixa o catalisador (no caso ESC nominal), perfazendo uma conversão de $87 \%$ dos óxidos de nitrogênio. Neste ponto, destacamos que a utilização de um catalisador de oxidação, como visto na figura 1, auxilia a conversão, pois sistemas SCR operam de forma mais eficiente no regime em que a relação entre monóxidos de nitrogênio e dióxidos de nitrogênio é de 1:1 (Schr et al., 2006).

Notamos também que, à medida que a razão $p_{2} / p_{1}$ diminui, o impacto da perturbação de $30 \%$ em $\mathrm{NO}_{\mathrm{x}, \text { in }}$ sobre $\mathrm{NO}_{\mathrm{x}, \text { out }}$ é menor. Ou seja, quanto maior o peso que damos ao termo de retroalimentação, mais robusto (i.e. menos sensível) é o sistema a variações na quantidade de óxidos de nitrogênio que entram no catalisador. Além disso, percebemos para o caso nominal uma leve diminuição na quantidade média de slip de amônia, à medida que $p_{2} / p_{1}$ diminui, o que pode ser interpretado como uma melhor utilização da amônia injetada e, portanto, eficiência do sistema de pós-tratamento. A emissão de óxidos de nitrogênio após o catalisador do sistema perturbado para o par $(43 ; 0,0)$, por exemplo, é $12 \%$ inferior àquela obtida para a técnica de controle em malha aberta. No entanto, as emissões em ambos os casos, quando $\mathrm{NO}_{\mathrm{x}, \text { in }}$ varia $30 \%$, ficam bem acima daquelas observadas no caso nominal.

A figura 3 ilustra a dinâmica das variáveis mais significativas do sistema de pós-tratamento no ciclo ESC, quando o par $(32 ; 2,0)$ é utilizado e $\mathrm{NO}_{\mathrm{x}, \text { in }}$ tem o valor nominal. Perceba que, no ciclo estático mostrado na figura 3 , os 
parâmetros do sistema (como $\mathrm{NO}_{\mathrm{x}, \mathrm{in}}, \mathrm{SV}$ e T) variam sobremaneira. Essa variação é especialmente severa para os parâmetros $k_{i}$ da equação (9), que dependem exponencialmente da temperatura, cuja variação é de mais de $200 \mathrm{~K}$, como mostrado na figura 3. A análise da figura 3 revela, ainda, que o slip de amônia atinge picos quando a temperatura dos gases de escape aumenta (comparar picos de slip com perfil da temperatura). Isso ocorre porque, ao aumento de $\mathrm{NO}_{\mathrm{x}, \text { in }}$, segue-se um aumento de $\mathrm{NO}_{\mathrm{x}, \text { out }}$, o que leva a uma maior ação de controle (i.e. maior injeção de amônia), como visto no gráfico de $u$, e consequente diminuição de $\mathrm{NO}_{\mathrm{x}, \text { out }}$. Esse aumento da ação de controle para fazer frente ao aumento de $\mathrm{NO}_{\mathrm{x}, \text { in }}$ acaba por adsorver mais amônia no interior do catalisador (ver gráfico de $\theta$ ) e, assim, tornar o catalisador mais suscetível à dessorção de amônia quando a temperatura aumenta.
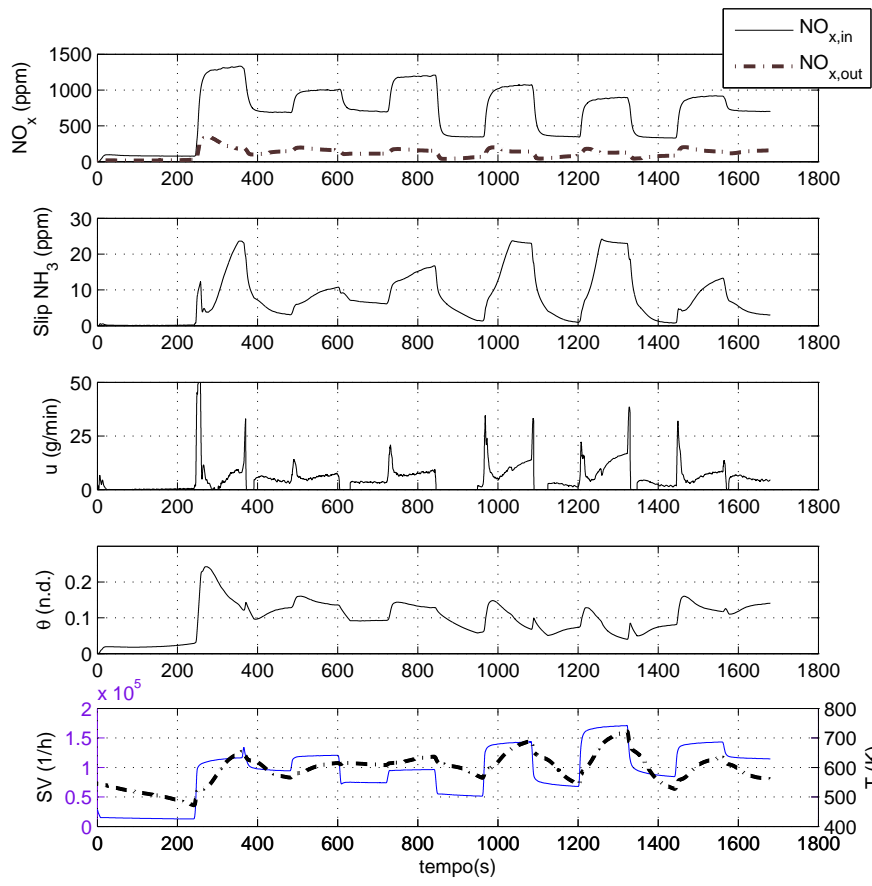

Figura 3: Dinâmica das variáveis mais significativas de um sis-

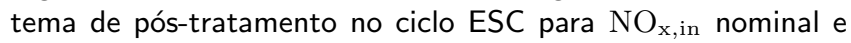
$\left(p_{1}, p_{2}\right)=(32 ; 2,0)$. No último gráfico, a linha tracejada corresponde à escala da temperatura, à direita. Layout da figura baseado no trabalho de Willems et al. (2007).

Na tabela 2, apresentamos também os resultados obtidos no ciclo ETC. Notemos que para o ciclo transiente, no caso em que a perturbação sobre $\mathrm{NO}_{\mathrm{x}, \text { in }}$ é de $30 \%$, $\mathrm{NO}_{\mathrm{x}, \text { out }}$ para o par $\left(p_{1}, p_{2}\right)=(43 ; 0,0)$ é $23 \%$ inferior àquela da técnica de controle em malha aberta. $\mathrm{O}$ ciclo ETC não é aqui mostrado, mas cabe ressaltar que o menor slip de amônia para o caso transiente, como se pode verificar comparando as tabelas 1 e 2 , deve-se às menores temperaturas alcançadas neste ciclo. Menor temperatura, menor a dessorção e, portanto, menor o slip. Como efeito colateral, no entanto, observamos a menor conversão de óxidos de nitrogênio $(8,08 \mathrm{~g} / \mathrm{kWh}$ são gerados pelo motor e 1,70 g/kWh, no mínimo, deixa o catalisador), chegando a um valor máximo de $79 \%$ para o caso nominal.

Tabela 2: Resultados da simulação no ciclo ETC. $\mathrm{NO}_{\mathrm{x}, \text { in }}$ no caso nominal é $8,08 \mathrm{~g} / \mathrm{kWh}$.

\begin{tabular}{cccc}
\hline \hline $\mathrm{NO}_{\mathrm{x}, \text { in }}$ & $\left(p_{1} ; p_{2}\right)$ & $\begin{array}{c}\mathrm{NO}_{\mathrm{x}, \text { out }} \\
{[\mathrm{g} / \mathrm{kWh}]}\end{array}$ & $\begin{array}{c}\text { slip } \mathrm{NH}_{3} \\
\text { médio } \\
{[\mathrm{ppm}]}\end{array}$ \\
\hline nominal & $\mathrm{MA}$ & 1,82 & 2,35 \\
& $(0,0 ; 7,5)$ & 1,80 & 2,39 \\
& $(8,2 ; 6,0)$ & 1,78 & 2,47 \\
& $(20 ; 4,0)$ & 1,75 & 2,64 \\
& $(32 ; 2,0)$ & 1,73 & 2,79 \\
$+30 \%$ & $(43 ; 0,0)$ & 1,70 & 2,89 \\
\hline & $(0,0 ; 7,5)$ & 2,97 & 1,15 \\
& $(8,2 ; 6,0)$ & 2,72 & 1,42 \\
& $(20 ; 4,0)$ & 2,52 & 1,81 \\
& $(32 ; 2,0)$ & 2,40 & 2,16 \\
& $(43 ; 0,0)$ & 2,32 & 2,44 \\
\hline
\end{tabular}

Por fim, o desempenho da lei de controle utilizada depende da convergência de $x_{4}$ para $\phi_{1}$ e $x_{3}$ para $\phi_{0}$. A figura 4 mostra a dinâmica de $x_{3}$ e $x_{4}$ para o ciclo ESC, no caso de $\mathrm{NO}_{\mathrm{x}, \text { in }}$ nominal e $\left(p_{1}, p_{2}\right)=(32 ; 2,0)$. A convergência de $x_{3}$ e $x_{4}$ para $\phi_{0}$ e $\phi_{1}$, respectivamente, é limitada especialmente por: a) taxa de decaimento de $x_{3}$, sobre a qual não temos controle, pois ela é função da cinética da reação de $x_{3}$ com os óxidos de nitrogênio ( $x_{3}$ acima de $\phi_{0}$ na figura 4 ); e b) fator multiplicativo $p_{x_{4}}$ de nossa lei de controle, que utilizamos para evitar altos picos de slip de amônia ( $x_{3}$ abaixo de $\phi_{0}$ na figura 4).

\subsection{Utilização de $\phi_{0}$ Linear por Partes}

O fato de uma perturbação de $30 \%$ em $\mathrm{NO}_{\mathrm{x}, \text { in }}$ ter levado

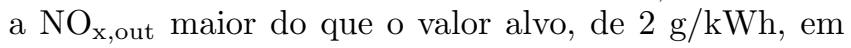
ambos os ciclos, motiva-nos a pensar em funções $\phi_{0}$ que aumentem a robustez de nosso controlador para o caso de variações nas concentrações de óxidos de nitrogênio emitidas pelo motor.

A função $\phi_{0}$ que utilizamos até aqui é linear em $\mathrm{NO}_{\mathrm{x}, \text { out }}$. Podemos obter melhores resultados, ao fazermos a magnitude de $\phi_{0}$ tanto maior, quanto mais $\mathrm{NO}_{\mathrm{x}, \text { out }}$ se afasta dos valores nominais. Uma possível candidata à nova $\phi_{0}$ 

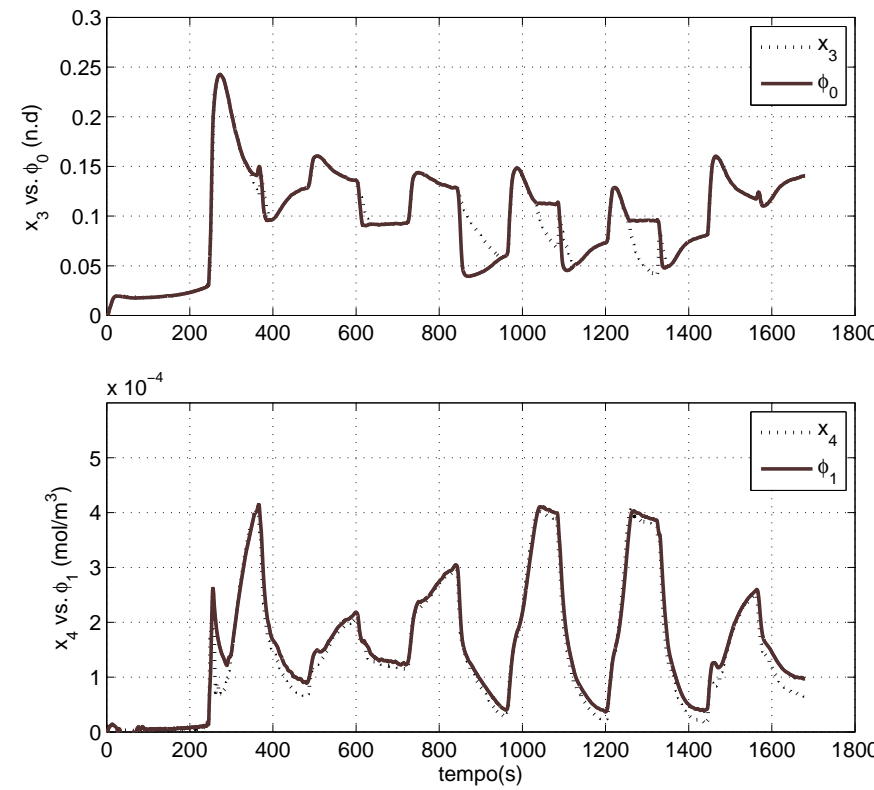

Figura 4: Dinâmica de $x_{3}$ e $x_{4}$ comparada àquela de $\phi_{0}$ e $\phi_{1}$ para um ciclo ESC.

é a função linear por partes que está ilustrada na figura 5. Notemos que essa função amplifica a atuação do controlador para $\mathrm{NO}_{\mathrm{x}, \text { out }}$ acima de $3 \times 10^{-3} \mathrm{~mol} / \mathrm{m}^{3}$. A configuração específica mostrada na figura 5 foi obtida por meio da análise do valor médio máximo de $\left(x_{1}+x_{2}\right)$, denotado por $\max \overline{\left(x_{1}+x_{2}\right)}$, tal que a emissão ainda fique dentro da janela permitida pela legislação. Desta forma, moldamos a função $\phi_{0}$ de tal forma que a inclinação da reta fosse maior quando $\left(x_{1}+x_{2}\right)$ fosse superior a $\max \overline{\left(x_{1}+x_{2}\right)}$. Assim, a lei de controle se utiliza de ganhos maiores, quanto maiores os desvios. Portanto, pode-se aumentar a conversão de óxidos de nitrogênio, mantendo o compromisso de reduzir o slip de amônia.

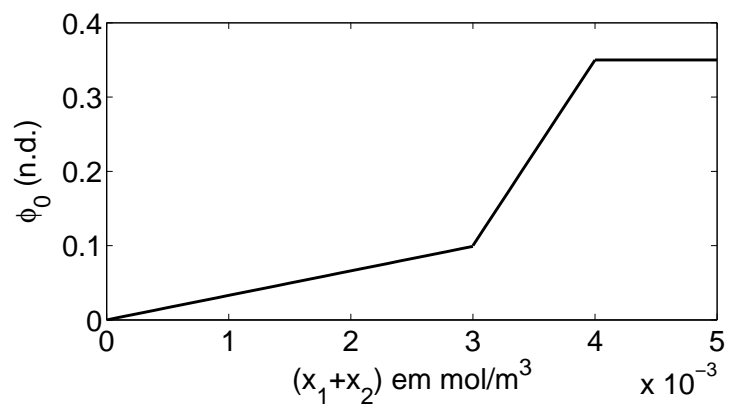

Figura 5: Nova função $\phi_{0}$ projetada para aumentar a robustez do sistema a variações em $\mathrm{NO}_{\mathrm{x}, \text { in }}$.

A tabela 3 mostra os resultados obtidos, quando utilizamos a lei de controle do tipo backstepping com $\phi_{0}$ como ilustrada na figura 5. A dinâmica do sistema não muda em relação àquilo que já foi apresentado na figura 3. Os resultados são melhores do que aqueles obtidos para a função $\phi_{0}$ anterior: $\mathrm{NO}_{\mathrm{x}, \text { out }}$, quando variamos $\mathrm{NO}_{\mathrm{x}, \text { in }}$ em $30 \%$, é de $1,94 \mathrm{~g} / \mathrm{kWh}, 21 \%$ inferior ao valor obtido para a lei de controle em malha aberta, satisfazendo a janela de $2 \mathrm{~g} / \mathrm{kWh}$ estipulada pela legislação EURO V. Resultados similares obtidos para a simulação do ciclo ETC estão descritos na tabela 4. É importante notar, no entanto, que os melhores resultados em termos de conversão de óxidos de nitrogênio levam a um aumento no slip de amônia. Fica evidente, assim, o compromisso que se estabelece em sistemas que operam com catalisadores SCR: mantido o hardware constante (i.e. catalisadores, motor, etc.), quanto maior a conversão, maior o slip de amônia.

Os melhores resultados obtidos com a nova $\phi_{0}$ mostram que uma análise mais aprofundada de diferentes funções pode melhorar o desempenho da lei de controle e incitam a continuidade deste estudo em busca de $\phi_{0}$ que minimize uma função objetivo, considerando: a) conversão de óxidos de nitrogênio; e b) redução de slip de amônia.

Tabela 3: Resultados da simulação no ciclo ESC para a nova $\phi_{0}$.

\begin{tabular}{ccc}
\hline \hline $\mathrm{NO}_{\mathrm{x}, \text { in }}$ & $\begin{array}{c}\mathrm{NO}_{\mathrm{x}, \text { out }} \\
{[\mathrm{g} / \mathrm{kWh}]}\end{array}$ & $\begin{array}{c}\text { slip } \mathrm{NH}_{3} \\
\text { médio } \\
{[\mathrm{ppm}]}\end{array}$ \\
\hline nominal & 1,60 & 7,99 \\
$30 \%$ & 1,94 & 9,33
\end{tabular}

Tabela 4: Resultados da simulação no ciclo ETC para a nova $\phi_{0}$.

\begin{tabular}{ccc}
\hline \hline $\mathrm{NO}_{\mathrm{x}, \text { in }}$ & $\begin{array}{c}\mathrm{NO}_{\mathrm{x}, \text { out }} \\
{[\mathrm{g} / \mathrm{kWh}]}\end{array}$ & $\begin{array}{c}\text { slip } \mathrm{NH}_{3} \\
\text { médio } \\
{[\mathrm{ppm}]}\end{array}$ \\
\hline nominal & 1,56 & 4,13 \\
$30 \%$ & 1,98 & 4,51 \\
\hline
\end{tabular}

\section{CONCLUSÃO}

Atingir os estritos limites legais para emissões de gases poluentes em veículos diesel é uma tarefa que exige uma combinação cuidadosa entre projeto e calibração de motor, juntamente com projeto e calibração do sistema de pós-tratamento. Os sistemas projetados precisam garantir não somente que os níveis de emissões de gases poluentes sejam baixos no momento da certificação do veículo, mas também que o impacto nas emissões desses gases seja o menor possível quando determinados parâ- 
metros do sistema variam ao longo do tempo. É especificamente nesse ponto, para garantir a robustez do sistema, que o projeto de controladores em malha fechada diferencia-se dos projetos comerciais atuais baseados no controle em malha aberta.

Mostramos nesse artigo, para o caso de perturbações simuladas de $30 \%$ sobre $\mathrm{NO}_{\mathrm{x}, \mathrm{in}}$, como o impacto sobre a concentração de $\mathrm{NO}_{\mathrm{x} \text {,out }}$ pode ser diminuído, tanto em ciclo estático, quanto em dinâmico, quando a quantidade de uréia injetada leva em consideração a concentração de óxidos de nitrogênio não convertidos que deixam o SCR. Dependendo da escolha da função $\phi_{0}$, o ponto de partida da estratégia backstepping, a quantidade de óxidos de nitrogênio que deixam o catalisador SCR pode ser $20 \%$ inferior àquela obtida quando uma técnica em malha aberta é utilizada, ou seja, obtemos uma diferença de $0,5 \mathrm{~g} / \mathrm{kWh}$ no ciclo ESC, o ciclo de testes estático. Tal redução é significativa, quando falamos de limites legais de $2 \mathrm{~g} / \mathrm{kWh}$ para o caso da legislação EURO V, por exemplo, que entrou em vigor no Brasil em 2012. Entretanto, esperávamos que a diferença entre as duas abordagens fosse ainda maior. Colaboraram para contrariar nossas previsões dois fatores: a) a conversão de óxidos de nitrogênio apresenta limitações físico-quimicas e estruturais, ou seja, a conversão é limitada não somente pela quantidade de uréia injetada, mas também pelo tamanho do catalisador, velocidade espacial e temperatura dos gases de escape, composição dos gases, etc., limites que não foram explorados neste estudo; e b) mesmo desprezando as limitações citadas em (a), não podemos responder às variações nos óxidos de nitrogênio com magnitudes de controle exageradas, pois estaríamos, desta forma, carregando o catalisador SCR com níveis de amônia facilmente sujeitos à dessorção e, portanto, muito slip em períodos de aumento de temperatura dos gases de escape.

Como próximos passos em nossos estudos, destacamos: a) o estudo formal de funções $\phi_{0}$ que otimizem o desempenho do controlador; e b) a implementação de um observador de estados para as variáveis $x_{3}$ e $x_{4}$, considerando como dado de entrada o sinal real gerado por sensores comerciais de $\mathrm{NO}_{\mathrm{x}}$, ou seja, a inclusão em nosso modelo da sensibilidade cruzada desses sensores à amônia. Trabalhos nesta direção já estão disponíveis na literatura, como por exemplo no artigo de Hsieh and Wang (2010).

\section{REFERÊNCIAS}

Chellaboina, V., Bhat, S. P., Haddad, W. M. and Bernstein, D. (2009). Modeling and analysis of massaction kinetics, IEEE Control Systems Magazine
29(4): $60-78$.

Chi, J. N. and DaCosta, H. (2005). Modeling and control of a urea-SCR aftertreatment system, SAE Technical Paper Series, Detroit. 2005-01-0966.

Devarakonda, M., Parker, G., Johnson, J., Strots, V. and Santhanam, S. (2008a). Adequacy of reduced order models for model-based control in a urea-SCR aftertreatment system, SAE Technical Paper Series, Detroit. 2008-01-0617.

Devarakonda, M., Parker, G., Johnson, J., Strots, V. and Santhanam, S. (2008b). Model-based estimation and control system development in a urea-SCR aftertreatment system, SAE Technical Paper Series, Detroit. 2008-01-1324.

Guenther, R. and Perondi, E. A. (2004). O controle em cascata de sistemas pneumticos de posicionamento, Revista SBA: Controle \& Automação 15(2): 149 161.

Guzzella, L. and Onder, C. (2004). Introduction to Modeling and Control of Internal Combustion Engine Systems, Springer.

Heywood, J. B. (1988). Internal Combustion Engine Fundamentals, McGraw-Hill Inc.

Hsieh, M. and Wang, J. (2010). Observer-based estimation of selective catalytic reduction catalyst ammonia storage, Journal of Automobile Engineering 224(9): 1199-1212.

Khalil, H. K. (2002). Nonlinear Systems - Third Edition, Prentice Hall.

Majewski, W. (2005). Selective catalytic reduction. www.dieselnet.com. Acessado em 14 de fevereiro de 2010 .

Pagano, D. J. (2007). Sntese de sistemas no-lineares, in L. A. Aguirre (ed.), Enciclopdia de Automtica Controle e Automao, Vol. 2, Edgard Blucher, chapter 7, pp. 148-172.

Raatz, T., Grieshaber, H., Wintrich, T. and Durst, M. (2005). Emissions Control Technology for Diesel Engines, Robert Bosch GmbH.

Ramirez, A., de Pieri, E. and Guenther, R. (2003). Controle em cascata de um manipulador robótico com um elo e uma transmissão flexível, Revista $S B A$ : Controle É Automação 14(4): 393 - 401. 
Schr, C. M., Onder, C. H. and Geering, H. P. (2006). Control of an SCR catalytic converter system for a mobile heavy-duty application, IEEE Transactions on Control Systems Technology 14(4): 641-653.

Seher, D., Reichelt, M. and Wickert, S. (2003). Control strategy for NOx - emission reduction with SCR, SAE Technical Paper Series, Texas. 2003-01-3362.

Upadhyay, D. and Nieuwstadt, M. V. (2006). Model based analysis and control design of a urea-SCR deNOx aftertreatment system, Journal of Dynamic Systems, Measurement and Control 128(3): 737741.

Wang, D., Yao, S., Shost, M., Yoo, J., Cabush, D., Racine, D., Cloudt, R. and Willems, F. (2008). Ammonia sensor for closed-loop SCR control, SAE Technical Paper Series, Detroit. 2008-01-0919.

Willems, F., Cloudt, R., van dem Eijnden, E., van Genderen, M. and Verbeek, R. (2007). Is closed-loop $\mathrm{SCR}$ control required to meet future emission targets?, SAE Technical Paper Series, Detroit. 2007$01-1574$. 